\title{
Lesão Cáustica por Ingestão de Ácido Nítrico em Lactente: Relato de Caso
}

\author{
Caustic Injury from Nitrous Acid Ingestion in Infants: Case Report \\ Lesión Cáustica por la Ingesta de Ácido Nítrico en el Lactante: Reporte de un Caso \\ José Antônio Chaves de Aguiar Filho ${ }^{1}$, Luísa Malheiro Ferreira ${ }^{2}$, Paula Dandara Correia de \\ Pinho $^{2}$, Lorena Vieira Gusmão ${ }^{2}$, Claudiojanes dos Reis ${ }^{3}$, Renato Neves Noronha ${ }^{4}$
}

\section{RESUMO}

Objetivo: Descrever caso raro e grave de ingestão de ácido nítrico por lactente, com ênfase na clínica, curso, manejo e prognóstico de acordo com as atualizações disponíveis na literatura. Detalhamentos de Caso: Lactente, vítima de intoxicação acidental por ingestão de grande volume de ácido nítrico, deu entrada no hospital com quadro de náuseas, vômitos e hematêmese, com presença de queimadura química em mucosa da cavidade bucal e faríngea. Evoluiu com queimadura esofagiana, perfuração gástrica e estenose de esôfago. Correções cirúrgicas foram necessárias para tratar as lesões, assim como seguimento com dilatações esofagianas. Considerações finais: $O$ relato demonstra a imprevisibilidade das lesões, além da necessidade de pesquisas especificas sobre o tratamento imediato e de longo prazo. A abordagem ao lactente mostrou-se eficaz devido ao diagnóstico precoce e terapêutica instituída de imediato. Há necessidade de educar o público, com ênfase na educação parental sobre os riscos da exposição de crianças a tais substâncias.

Palavras-chave: Envenenamento, Ácido Nítrico, Caústicos.

\begin{abstract}
Objective: To describe a rare and serious case of nitric acid ingestion by an infant, with emphasis on clinical evaluation, course, management and prognosis, according to the latest updates about the issue. Case Details: Infant, victim of caustic intoxication due to accidental ingestion of a great volume of nitric acid, was admitted to the hospital presenting nausea, vomiting and hematemesis, with the presence of chemical burn in the mucosa of the oral and pharyngeal cavity. The patient evolution occurred with esophageal burn, gastric perforation and esophagus stenosis. Surgical corrections where necessary to treat the existent lesions, as well as follow up with esophageal dilatations. Final considerations: The case shows the unpredictability of the lesions, as well as the necessity of researching new specific immediate and long-term treatments. The approach of the infant has been shown as effective, due to the early diagnosis and the immediate therapy instituted. There's also a need to educate the population, with emphasis on parental education about the risks of exposing children to such substances.
\end{abstract}

Key words: Poisoning, Nitric Acid, Caustics.

\section{RESUMEN}

Objetivo: Describir un caso raro y severo de la ingesta de lo ácido nítrico en uno infante, con énfasis en la clínica, el curso, el manejo y lo pronóstico. Detalles del caso: El bebé, víctima de envenenamiento accidental por la ingestión de gran cantidad de lo ácido nítrico, ingresó en el hospital con náuseas, vómitos y hematemesis, con quemaduras químicas en la mucosa de la cavidad oral y faríngea. Evolucionó con quemaduras esofágicas, perforación gástrica y estenosis esofágica. Las correcciones quirúrgicas fueron necesarias para tratar las lesiones, así como seguimiento con dilataciones esofágicas. Consideraciones finales: Este informe de caso demuestra la imprevisibilidad de las lesiones, así como la necesidad de

\footnotetext{
1 Universidade Federal de Minas Gerais, Montes Claros - Minas Gerais. *E-mail: juniormed7@ @otmail.com

2 Faculdades Unidas do Norte de Minas, Montes Claros - Minas Gerais.

3 Universidade Estadual de Montes Claros, Montes Claros - Minas Gerais.

${ }^{4}$ Hospital Santa Marcelina, São Paulo - São Paulo.
} 
investigaciones específicas de nuevos tratamientos inmediatos ya largo plazo. El enfoque del infante demostró ser efectivo debido al diagnóstico temprano y la terapia instituida inmediatamente. Existe la necesidad de educar al público, con énfasis en la crianza de los hijos sobre los riesgos de exponer a los niños a tales sustancias.

Palabras clave: Envenenamiento, Ácido nítrico, Cáusticos.

\section{INTRODUÇÃO}

As intoxicações ou envenenamentos são causados pela ingestão, inalação ou exposição a alguma substância tóxica (nociva) ao organismo que pode provocar sequelas e até mesmo a morte se o indivíduo não for socorrido a tempo. O ambiente domiciliar apresenta uma grande variedade de agentes como plantas tóxicas, medicamentos, pesticidas, produtos de limpeza e higiene que, quando não armazenados ou utilizados de forma incorreta, representam risco para intoxicação e envenenamento, principalmente em crianças (UNIVERSIDADE FEDERAL DE MINAS GERAIS, 2006).

Segundo o Ministério da Saúde (2014), no Brasil, de agosto de 2013 a agosto de 2014, ocorreram 1437 internações devido a envenenamento ou intoxicação acidental por exposição a substâncias nocivas na faixa etária de 0 a 24 anos. Estas internações ocasionaram um gasto de quase 500 mil reais para o Sistema Único de Saúde.

Os dados epidemiológicos sobre incidência de lesão gástrica por ingestão de substâncias corrosivas são considerados escassos devido à subnotificação, mas sabe-se que crianças entre 2 e 6 anos de idade representam até $80 \%$ das ingestões, que são acidentais e normalmente acarretam em lesões leves a moderadas, enquanto a outra parcela de casos é de ingestão por adultos, em sua maioria entre os 30 aos 40 anos de idade, que tem intenções suicidas e, portanto, tendem a apresentar ocorrências mais graves (CHIRICA M, et al, 2017).

Os ácidos geralmente acometem o estômago poupando o esôfago, e causam inflamação e necrose de coagulação da mucosa. A extensão da lesão depende das especificidades da ingestão (volume, concentração e natureza do ácido), do conteúdo gástrico no momento da ingestão e da duração do contato do material com a mucosa gástrica. Após a ingestão ácida, os pacientes podem apresentar-se assintomáticos ou uma combinação de sintomas, entre eles: dispneia, disfagia, odinofagia, náuseas, êmese recorrente, hematêmese, sialorreia, dor ou rigidez abdominal e dor torácica. As complicações precoces incluem ulceração, sangramento e perfuração; e as tardias são fibrose e obstrução da saída gástrica (LUPA M et al, 2009).

Após garantir uma via aérea segura e definitiva, deve-se realizar um exame físico minucioso e uma história completa, com atenção especial para o momento da ingestão, identidade do agente e quantidade ingerida. Exames a serem realizados incluem: provas laboratoriais, radiografias de tórax e abdome, Endoscopia Digestiva Alta (EDA) - padrão-ouro para o diagnóstico de lesões do trato gastrointestinal superior (TGIS) -, e Tomografia Computadorizada (TC). O tratamento consiste na correção de distúrbios metabólicos e coagulopatias (LUPA M, et al, 2009).

Diante disso o objetivo deste trabalho foi descrever um caso raro e grave de ingestão de ácido nítrico em uma criança de um ano de idade, que desenvolveu necrose esofágica e perfuração gástrica, enfatizando a clínica, curso, manejo e prognóstico a partir da literatura atual.

\section{RELATO DE EXPERIÊNCIA}

Paciente do sexo masculino, caucasiano, de 1 ano e 6 meses de idade, deu entrada no Serviço de Emergência no Sistema Único de Saúde (SUS)/MG, com história clínica de ingestão acidental de Ácido Nítrico enquanto o lactente brincava sozinho no curral de sua casa, em uma fazenda na zona rural, apresentando também náuseas, vômitos e três episódios de hematêmese. A acompanhante, mãe do paciente, negava comorbidades e alergias. Ao exame clínico, lactente apresentava queimadura química em toda a superfície mucosa da cavidade bucal e faríngea, além de sialorreia moderada. Estava corado, hidratado, acianótico, anictérico, sem edemas. 
Foi iniciada monitorização contínua dos dados vitais com monitor cardíaco, solicitadas vaga em Centro de Terapia Intensiva (CTI), avaliações otorrinolaringológica e da cirurgia pediátrica, além de Endoscopia Digestiva Alta (EDA). Foi iniciado esquema antibiótico associando Ceftriaxona e Metronidazol, e realizada avaliação da anestesiologia para a realização de intubação orotraqueal (IOT) caso o paciente evoluísse com edema de cordas vocais. Cuidados clínicos foram mantidos e envolviam dieta suspensa, analgesia e soroterapia. Após 12-24 horas da admissão, o lactente evoluiu com peritonite, foi encaminhado para realização de EDA (Figura 1 A), a qual evidenciou lesão extensa em todo o esôfago e estômago, com perfuração de parede gástrica (Figura 1 B).

Figura 1 - A. Visão endoscópica da mucosa esofágica 24h após lesão mostra queimadura de grau 3b, o que significa presença de necrose extensa. Observe a perda da coloração esofágica habitual (avermelhada) e as áreas extensas de descoloração (necrose); B. Aparelho passando pela perfuração provocada pelo ácido na parede anterior do estômago, com visão direta do grande omento (indicado pela seta) na cavidade abdominal.

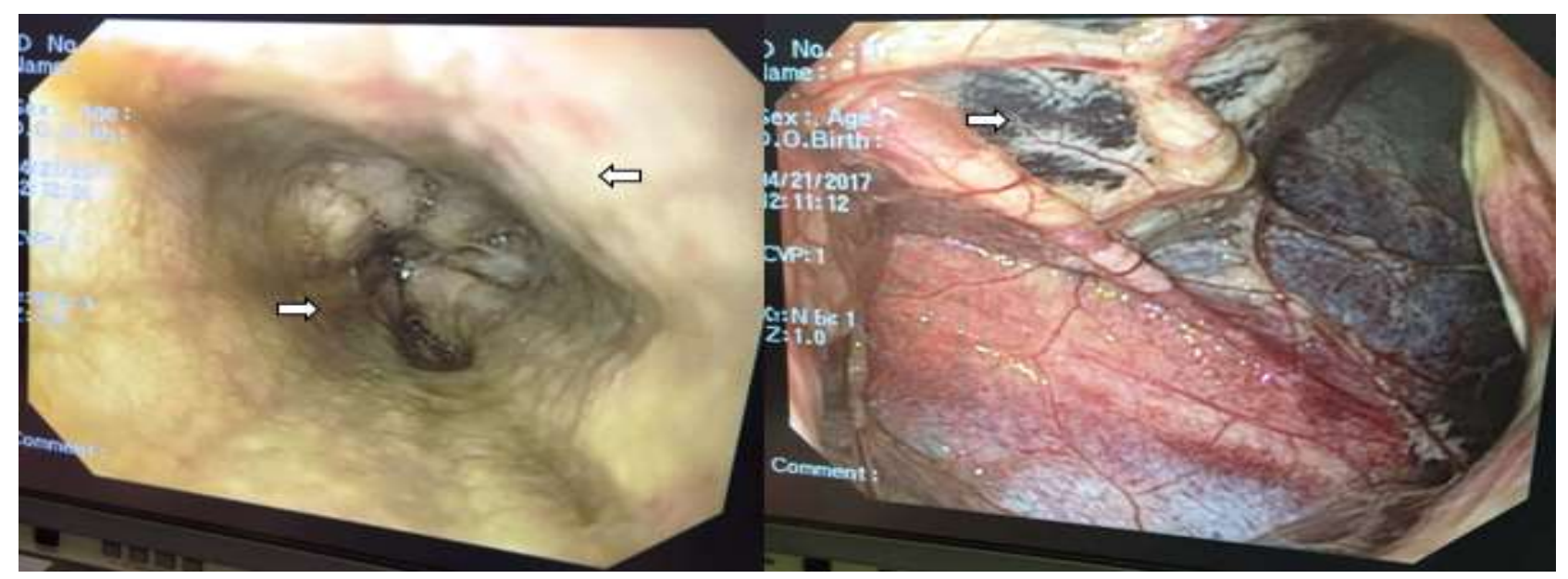

Fonte: Aguiar Filho JAC, Ferreira LM, Pinho PDC, et al., 2019.

Foi encaminhado ao Bloco Cirúrgico, onde foi visualizado edema importante em pregas vocais e realizada laparotomia exploratória de emergência. Esta identificou perfuração e necrose em grande curvatura gástrica (Figura 2).

Figura 2 - Imagem obtida após laparotomia e abertura da cavidade abdominal. Com a exposição do estômago, nota-se extensa área de necrose com perfuração (indicado pela seta) na parede anterior do estômago, próximo ao piloro.

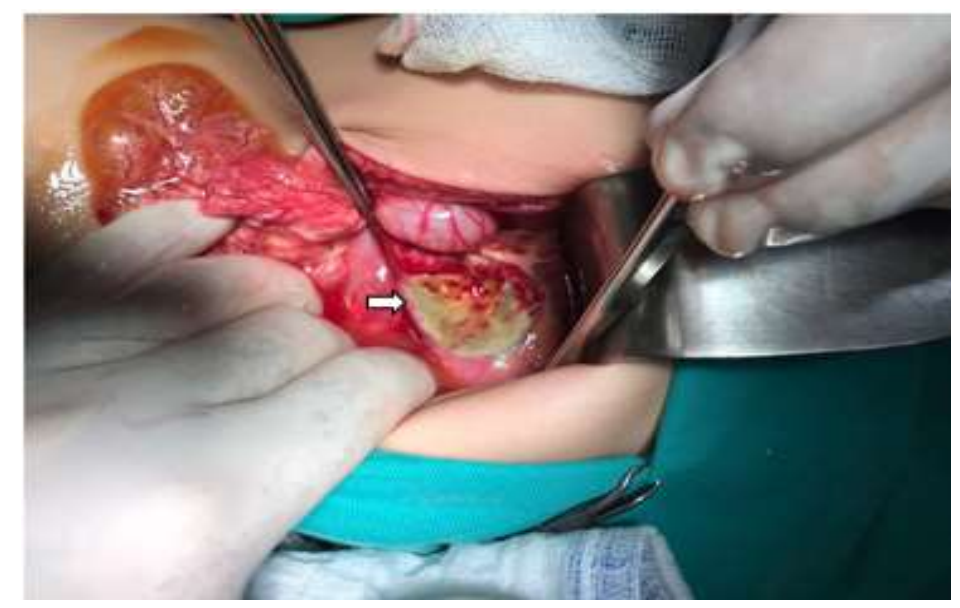

Fonte: Aguiar Filho JAC, Ferreira LM, Pinho PDC, et al., 2019. 
Foi realizado uma gastrectomia parcial e gastrostomia, sem intercorrências (Figura 3). A propedêutica pós operatória foi: manter criança em sedação contínua com Midazolan $15 \mathrm{mg} / 3 \mathrm{~mL}$ na dose de $2 \mathrm{mcg} / \mathrm{kg} / \mathrm{min}$ a $1 \mathrm{~mL} / \mathrm{h}$ e Fentanil $0,05 \mathrm{mg} / \mathrm{mL}$ na dose de $3 \mathrm{mcg} / \mathrm{kg} / \mathrm{h}$ a $2 \mathrm{~mL} / \mathrm{h}$, respirando em ventilação mecânica (VM) por tubo orotraqueal; iniciar dieta parenteral, terapia com Inibidor de Bomba de $\mathrm{H}_{+}$e esquema de antibioticoterapia para Pneumonite Química com Clindamicina $150 \mathrm{mg} / \mathrm{kg} / \mathrm{dia}$, manter sonda de gastrostomia aberta, além de cuidados clínicos com a pediatria.

Figura 3 - Imagem evidenciando a reconstrução do estômago (indicado pela seta) após gastrectomia parcial com a retirada do antro gástrico.

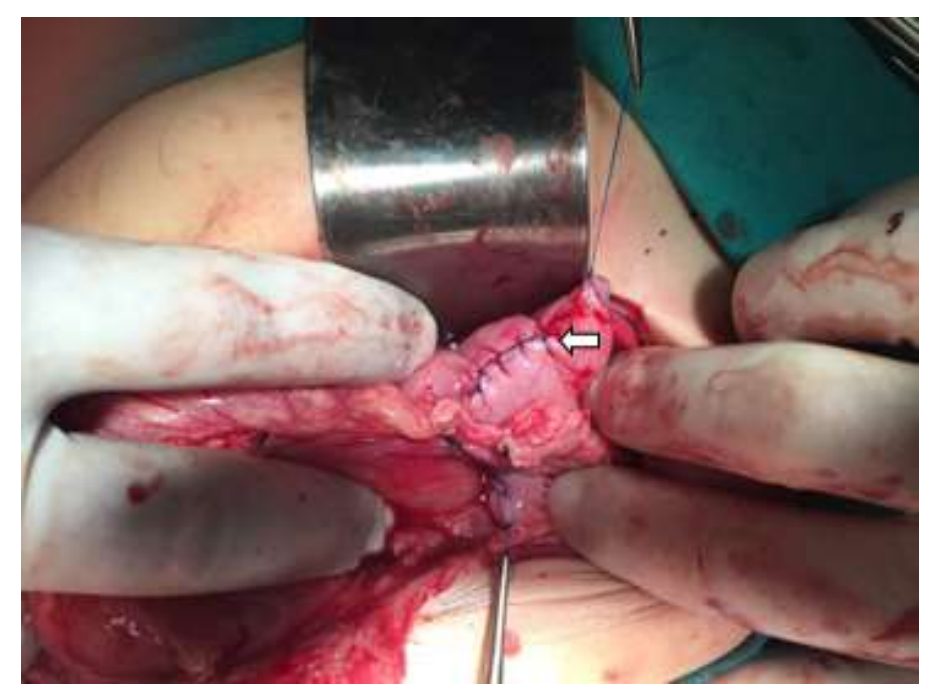

Fonte: Aguiar Filho JAC, Ferreira LM, Pinho PDC, et al., 2019.

Lactente evoluiu em estado grave com sucessivas intercorrências: queda da Hemoglobina no pósoperatório por sangramento durante o procedimento cirúrgico (realizada transfusão de concentrado de hemácias $15 \mathrm{~mL} / \mathrm{kg}$ ), picos febris evidenciando sepse e quedas de saturação. O paciente foi transferido para o CTI com sepse grave, onde permaneceu por vinte dias em terapia com Inibidor de Bomba de $\mathrm{H}+$ e esquema antibiótico de amplo espectro para Pneumonite Química (com Ceftriaxona 100 $\mathrm{mg} / \mathrm{kg} / \mathrm{dia}$ e Clindamicina $150 \mathrm{mg} / \mathrm{kg} / \mathrm{dia}$ ) além de terapia de suporte com hidratação, analgesia e antieméticos se necessário, até receber alta, estando hemodinamicamente estável, respirando em ar ambiente e mantendo sonda de gastrostomia. Orientação de acompanhamento mensal com cirurgião pediátrico e ultrassonografista. No primeiro deles, identificou-se a presença de complicação - estenose de esôfago - que implicou na realização de dilatações, ainda mantidas até seis meses após a alta.

Atualmente, a criança ingere alimentos por via oral, está mantendo o peso, já não possui sonda de gastrostomia e continua realizando dilatações endoscópicas conforme orientação médica.

\section{DISCUSSÃO}

A ingestão corrosiva é um evento raro, mas potencialmente devastador e, apesar da disponibilidade de estratégias preventivas de saúde, as lesões continuam a ocorrer, o que é creditado ao descuido dos cuidadores e ao armazenamento inadequado nos casos com crianças e à tentativa deliberada de autoextermínio entre adultos. As ingestões variam de casos leves sem lesões graves à necrose total do esôfago e estômago, que dependem grandemente do mecanismo de lesão do agente ingerido, da quantidade ingerida, da concentração e de características individuais do corpo (NIEDZIELSKI A, et al, 2019). A baixa incidência de lesões corrosivas faz com que os clínicos em geral tenham experiência limitada com esse tópico, e a ausência de diretrizes baseadas em evidências faz com que a incerteza sobre a melhor prática clínica 
persista, o que é acarreta em variações significativas no gerenciamento de pacientes (CHIRICA M, et al, 2017).

A idade de ocorrência das ingestões mostra padrão bimodal, com primeiro pico em crianças com até 5 anos de idade, que representam $80 \%$ das lesões por ingestão e cujos fatores de risco associado incluem gênero masculino, pais com tempo menor de educação, transtorno de déficit de atenção/hiperatividade, idade materna jovem, falta de supervisão parental e moradia rural (MARION A, NOMANOGLU A, 2017). A outra idade comum é entre os adultos com idades entre 21 anos ou mais. A maioria das ingestões observadas nesta população são tentativas de suicídio, e cujos número vêm aumentando especialmente em países de baixa renda, associados à falta de regulamentação e programas efetivos de prevenção e saúde pública. $A$ ingestão de ácido é mais comum em países asiáticos, onde essas substâncias são relativamente baratas e facilmente disponíveis, o que pode facilitar esse tipo de acidente (SCHAFFER SB, HEBERT AF, 2000).

O paciente em estudo enquadra-se no perfil de ingestão acidental, em zona rural, de aspecto social frágil, em país subdesenvolvido, no qual projetos de educação e prevenção em saúde são escassos (FINKELMAN J, 2002). Houve ingestão volumosa de Ácido Nítrico, solução classificada como oxidante, corrosiva e muito tóxica, que pode ser encontrada em limpadores de vasos sanitários (UNIVERSIDADE FEDERAL DE MINAS GERAIS, 2006). No caso relatado, a substância estava contida em produto de higienização de currais.

Os ácidos induzem a necrose de coagulação, sendo que nas primeiras 24 horas predominam hemorragia, trombose, inflamação e edema, com formação de escaras pela consolidação do tecido conjuntivo, o que podem limitar a penetração no tecido, o que não parece ser a principal variável na determinação da extensão da lesão. Além disso, esse fato reafirma a raridade do caso descrito, no qual verificou-se perfuração da parede gástrica, e torna possível hipotetizar que tenha ocorrido uma incapacidade da secreção gástrica em neutralizar a substância ingerida, o que poderia ter contribuído para o rompimento da mucosa do antro gástrico. Os ácidos geralmente acometem o estômago, poupando o esôfago em cerca de $80 \%$ dos casos (CHIRICA M, 2017). As razões pelas quais o esôfago seria poupado de lesões incluem trânsito rápido pelo canal e seu aspecto histológico: a célula escamosa estratificada do epitélio esofágico é espessa e relativamente ácidoresistente, em comparação com a fina mucosa colunar gástrica (LUPA M et al, 2009). No caso relatado, o esôfago não foi poupado.

O dano tecidual significativo ocorre em segundos de ingestão de agentes corrosivos fortes. Hemorragia, trombose e inflamação com edema são processos dominantes durante as primeiras 24 horas após a ingestão. Queimaduras graves podem avançar para áreas focais de necrose, com perfuração à medida que a inflamação se estende através de camadas musculares, acarretando trombose submucosa e invasão bacteriana (BONAVINA L et al, 2015). É possível que a extensa lesão desenvolvida neste caso se deva ao fato de que uma grande quantidade de corrosivo concentrado foi ingerida em um estômago vazio.

Os pacientes com ingestão ácida podem estar assintomáticos ou apresentar uma série de sintomas, incluindo: dor epigástrica, retroesternal e orofaríngea. A presença de dispneia, rouquidão e estridor sugere 0 envolvimento da laringe e requerem investigação laringoscópica. Disfagia, odinofagia e sialorreia sugerem danos esofágicos, enquanto vômito, hematêmese e dor abdominal podem sugerir danos gástricos. Dor contínua, peritonite, taquicardia, leucocitose prolongada e acidose levantam a suspeita de perfuração. Contudo, a sintomatologia não se correlaciona em absoluto com a extensão das lesões (KLUGER Y et al, 2017).

No presente relato o lactente apresentou úlceras em cavidade oral, hematêmese, náuseas, vômitos e sialorreia. Este caso é singular pela ocorrência de necrose esofágica e gástrica extensas, além de edema agudo da laringe com necessidade de intubação orotraqueal. Após ingestão maciça, testes de laboratório de emergência devem ser feitos, incluindo hemograma completo, $\mathrm{pH}$ e lactato sérico, concentrações séricas de sódio, potássio, cloro, ureia, creatinina, aspartato aminotransferase, alanina aminotransferase e bilirrubina. Resultados de laboratório normais não excluem necrose transmural. Leucocitose, alta concentração sérica de proteína $\mathrm{C}$ reativa, $\mathrm{pH}$ baixo, concentração elevada de lactato no sangue, insuficiência renal, testes de função hepática alterados e trombocitopenia podem prever a necrose transmural. (CHIRICA M et al, 2015). 
A endoscopia é o exame mais efetivo para determinar a extensão das lesões e direcionar o tratamento (NIEDZIELSKY et al, 2017). Há concordância de que é mais bem realizada entre 12-48 horas; se realizada precocemente pode perder lesões evolutivas, enquanto a tardia pode gerar perfuração. A classificação endoscópica de Zargar possui ampla aceitação traz: grau 0 como normal; grau 1 edema e hiperemia da mucosa; grau 2a ulcerações superficiais localizadas e friáveis; grau $2 b$ ulcerações circunferenciais e profundas; grau 3a são ulcerações múltiplas e profundas e pequenas áreas de necrose; e 3b como extensa necrose (CHIRICA et al, 2017). O paciente relatado foi submetido a esofagoscopia com endoscópio flexível 12-24h após a ingestão, e a lesão foi classificada como 3b, com extensa necrose em esôfago e estômago.

O consenso produzido na conferência de cirurgia do World Society of Emergency informou que, embora a endoscopia seja a pedra angular da gestão de lesões cáusticas, o uso da TC ajuda a atenuar deficiências dos algoritmos baseados em endoscopia, como sobre o dano transmural das paredes esofágica e gástrica e a extensão da necrose (BONAVINA L et al, 2015).

O uso de antibióticos, esteroides e terapia antirefluxo foram os outros focos da gestão. Na literatura, há divergências sobre o uso desses medicamentos. Os defensores se baseiam em estudos que indicam que uso de antibióticos diminuiria a estenose posterior por diminuir a inflamação local ao diminuir a microbiota e a inflamação, enquanto os esteroides diminuiriam a formação de colágeno e diminuiriam a incidência de estenose. Entretanto, os estudos são controversos, assim como a opinião da classe médica sobre a melhor abordagem (KLUGER Y et al, 2017).

Recomenda-se o uso rotineiro de terapia antirrefluxo a fim de evitar lesões esofágicas secundárias associadas ao refluxo que pode atuar de forma sinérgica para novos danos; entretanto, não existem estudos controlados com crianças que tenham sido capazes de confirmar a eficácia dessa abordagem (SHUB M, 2015). A maioria das recomendações sobre a prevenção e o tratamento baseiam-se em pequenos estudos retrospectivos, havendo a necessidade de estudos sobre este tópico.

A dieta deve ser suspensa e a jejunostomia alimentadora é uma excelente rota para nutrição, excluindo o trato digestivo superior, aguardando a cura da mucosa. A nutrição parenteral total também pode ser indicada para esses pacientes, inclusive para aqueles debilitados, como forma de correção de distúrbios hidroeletrolíticos antes da jejunostomia ou do tratamento definitivo. Não há consenso sobre o intervalo ideal antes deste (ANANTHAKRISHMAN N et al, 2010).

Em alguns pacientes, a intervenção cirúrgica é necessária. A presença de necrose extensa à endoscopia e evidência de perfuração são indicações para intervenção imediata. Tal conduta foi adotada no presente relato, pois, após identificada perfuração à EDA, o lactente seguiu de imediato para ato operatório. A laparotomia é a cirurgia de emergência padrão, mas a exploração laparoscópica de sucesso foi relatada. Esofagectomia, esofagogastrectomia ou gastrectomia podem ser indicados se a necrose extensa for confirmada (DAPRI $G$ et al, 2007). A criança deste caso foi submetida a gastrectomia parcial, sem intercorrências.

A gastrectomia em crianças é mais problemática do que em adultos devido a efeitos colaterais como anemia, deficiência de vitamina B12 e retardo do crescimento (BONAVINA $L$ et al, 2015). A anemia desenvolvida no caso descrito, com necessidade de hemotransfusão, possivelmente teve como etiologia 0 sangramento do ato operatório. Contudo, anemias de etiologias carenciais ou deficiência de vitamina B12 não foram observadas em sua evolução, visto que a liberação da dieta foi realizada precocemente no pósoperatório, devido à confecção de gastrostomia. Faz-se necessário o acompanhamento do padrão de crescimento dessa criança, visto que a ocorrência do fato é precoce para tal avaliação.

Sequelas tardias de ingestão ácida ocorrem após períodos variáveis, são incapacitantes e podem ser fatais, e a formação de estenose é a mais comum (SHUB M, 2015). No caso em destaque, o paciente evoluiu com estenose esofágica. Estas, geralmente, desenvolvem-se dentro de 2 meses ( 3 semanas a 1 ano) após a ingestão e pode ser prevista, de forma confiável, tanto por EDA quanto por TC. A dilatação endoscópica é a opção de gerenciamento de primeira linha (BONAVINA $L$ et al, 2015). Dados observacionais indicam que essas dilatações devam ser realizadas a cada uma a três semanas até a alimentação oral ser tolerada, e que 
após isso a realização de novas sessões deva ser regulada com base na recorrência da sintomatologia (SHUB M, 2015).

A ingestão de substância caustica é associada a risco aumentado de câncer esofágico, com ocorrência 1000 vezes maior do que a esperada para a população em geral tanto do desenvolvimento de adenocarcinoma quanto para o carcinoma de células escamosas (SHUB M, 2015).

Programas de saúde pública para educar o público e estabelecer medidas efetivas que limitem o acesso a agentes corrosivos fortes é fundamental para diminuir a incidência e gravidade da ingestão. A alta taxa de complicações enfatiza a necessidade de um acompanhamento multidisciplinar a longo prazo.

\section{CONSIDERAÇÕES FINAIS}

O estudo evidenciou o caso de lactente com lesão cáustica pela ingestão de ácido nítrico que evoluiu com complicações, e eventual quadro estável. Vê-se que a intoxicação por ácido nítrico apresenta natureza imprevisível. A epidemiologia e fisiopatologia da ingestão dessa substância têm sido pouco descritas na literatura, e a quantidade reduzida de estudos traz dificuldade às equipes médicas no momento de lidar com as lesões e complicações. O tratamento da ingestão cáustica deve ser individualizado, dando atenção até a lesões de baixo grau, frente às possíveis complicações. É preciso educar o público com campanhas dirigidas especificamente aos cuidadores de crianças pequenas, enfatizando que elas não devem ter acesso a corrosivos. As opções atuais de tratamentos visam tratar as lesões imediatas e prevenir complicações futuras.

\section{REFERÊNCIAS}

1. ANANTHAKRISHMAN N, et al. Chronic corrosive injuries of the stomach: a single unit experience of 109 patientsover thirty years. World J Surg 2010 [DOI: 1007/s00268-010-0393-6 published on line 23 January 2010]

2. BONAVINA L, et al. Foregut caustic injuries: results of the world society of emergency surgery consensus conference. World J Emerg Surg 2015; 10: 44.

3. CHIRICA M, et al. Caustic ingestion. The Lancet, 2017: 389: 2041-52.

4. CHIRICA M, et al. Computed tomography evaluation of high-grade esophageal necrosis after corrosive ingestion to avoid unnecessary esophagectomy. Surg Endosc 2015; 29: 1452-61.

5. DRAPRI G, et al. Laparoscopic transhiatal esophago-gastrectomy after corrosive injury. Surg Endosc 2007; 21: 232225

6. FINKELMAN J. Caminhos da saúde no Brasil. Rio de Janeiro: Editora FIOCRUZ, 2002. 328

7. Informações em Saúde: Indicadores de morbidade e mortalidade. In.: DATASUS. Brasília. Disponível em: http://www.datasus.gov.br. Acesso em: 14 out. 2014.

8. KIM YT, et al. Is it necessary to resect the diseased esophagus in performing reconstruction for corrosive esophageal stricture? European Journal of Cardiothoracic Surgery, 2001; 20: 1-6.

9. KLUGER Y, et al. Caustic ingestion management: world society of emergency surgery preliminary survey of expert opinion. World J Emerg Surg. 2015; 10:48

10. KREY H. Treatment of corrosive lesions in the esophagus. Acta Otolaryngol 1952; 102: 1-49.

11. LUPA M, et al. Update on the diagnosis and treatment of caustic ingestion. Ochsner J 2009; 9: 54-9.

12. MARION A, NUMANOGLU A. Caustic ingestion in children - a review. Pediatr Surg. 2017;

13. NIEDZIELSKI A, et al. Caustic agents ingestion in children: a 51-year retrospective cohort study. Ear, Nose \& Throat J. 2019. 1-6

14. SCHAFFER SB, HEBERT AF. Ingestão cáustica. J La State Med Soc. 2000; 152 (12): 590-596.

15. SHUB MD. Therapy of caustic ingestion: new treatment considerations. Peadiatr. 2015; $27: 5$.

16. UNIVERSIDADE FEDERAL DE MINAS GERAIS. Faculdade de Farmácia. Departamento de Análises Clínicas e Toxicológicas. Noções básicas de toxicologia. Belo Horizonte: 2006; $51 \mathrm{p}$. 